\title{
A NOVEL SYNTHESIS OF 2'-HYDROXY-1',3'-XYLYL CROWN ETHERS
}

\author{
M. van der LeiJ, H. J. OOSTERINK, R. H. HALl and D. N. ReInHOUdT* \\ Laboratory of Organic Chemistry, Twente University of Technology, Enschede, The Netherlands
}

(Received in UK 6 April 1981)

\begin{abstract}
Six novel $2^{\prime}$ - hydroxy $-1^{\prime}, 3^{\prime}$ - xylyl crown ethers $(8 a+\text { and } 13)^{1}$ have been synthesized utilizing the allyl group to protect the $\mathrm{OH}$ function during the cyclization reaction. The macrocycles $6 \mathrm{a} e$ were formed in yields of 26 to $52 \%$, by intermolecular reaction of 4 - chloro - 2,6 - bis(bromomethyl) - 1 - (2 - propenyloxy)benzene (5) with polyethylene glycols; $6 \mathrm{a}$ was also obtained by an intramolecular cyclization reaction of monotosylate 14.

A 30 -membered ring with a $2^{\prime}$ - hydroxy $-1^{\prime}, 3^{\prime}$ - xylyl sub-unit was obtained in $87 \%$ yield by reaction of ditosylate 9 with bis [2 - (o - hydroxyphenoxy)ethyl]ether (11) in the presence of cesium fluoride. The synthesis of crown ethers with a $2^{\prime}$ - hydroxy - 1',3' - xylyl sub-unit (1ce, $\mathrm{H}$ for $\mathrm{CH}_{3}$ ) by demethylation of the corresponding $2^{\prime}$-methoxy crown ethers lc-e with lithium iodide were unsuccessful; it would appear that the demethylation reaction is restricted to 15 - and 18 -membered rings. One of the $2^{\prime}$ - hydroxy - $1^{\prime}, 3^{\prime}$ - xylyl crown ethers $8 \mathrm{~d}$ forms a crystalline 1:1-complex with water.
\end{abstract}

Proton transfer reactions in crown ether complexes have not been studied in detail. A complex of a chiral 2,2' bis(carboxymethoxymethyl) - 1,1 - binapthyl crown ether with an amino acid in which one of the acid groups dunates a proton to the amino group of the amino acid ${ }^{3}$ and a crystalline complex of $2^{\prime}$ - carboxy - $1^{\prime}, 3^{\prime}$ - xylyl 18 - crown -5 and t-butylamine ${ }^{4.5}$ have been described. The complexation of $5^{\prime}$ - nitro - $2^{\prime}$ - hydroxy - 1',3' - xylyl crown ethers and ammonia ${ }^{6}$ might also involve a proton transfer process.

In relation with our work on the complexation of urea? we are currently investigating proton transfer reactions in complexes of crown ethers with neutral molecules. For this work crown ethers of various ringsizes with intraannularly placed acid groups are required. $\dagger$ Since the $1^{\prime}, 3^{\prime}$-xylyl sub-unit provides the possibility of various substituents at the $2^{\prime}$-position in such a way that they point towards the crown ether cavity, we decided to investigate preparative routes to such crown ethers with $\mathrm{OH}$ functions at the 2 -position.

The preparation of several crown ethers with $1^{\prime}, 3^{\prime}$. xylyl subunits and with additional functional groups either at the intraannular 2 -position or at the more remote $4^{\prime}$ - or $5^{\prime}$-position have been described $d^{4,10-13}$ and the mode of substitution has been shown to effect the complexing properties with alkali metal and ammonium salts. ${ }^{411.14}$ Substitution at the $2^{\prime}$-position generally decreases the stability of the complexes with ammonium salts and this has been attributed to crowding of the crown ether cavity. ${ }^{4.10}$ Substituents at the $5^{\prime}$-position have a considerable effect on complexation showing that the aryl ring is involved in this process."

The preparation of $2^{\prime}$ - hydroxy - $1^{\prime}, 3^{\prime}$ - xylyl crown ethers has been reported previously by two groups. In both cases the major problem in this synthesis was to find a suitable protecting group for the $\mathrm{OH}$ function that should be stable under the strongly basic conditions of the Williamson ether synthesis and that could be

tCPK molecular models indicate that ringsizes of at least 27 atoms are required to encapsulate small organic polyfunctional molecules or ions and although full information about the structure is not available, complexation of guanidinium salts by benzo - 27 - crown - 9 supports this assumption. ${ }^{8.9}$ removed selectively under conditions which do not cleave the benzylic ether bonds. Koenig et al. ${ }^{15}$ reported a synthesis of $5^{\prime}$ - methyl $-2^{\prime}$ - hydroxy $-1^{\prime}, 3^{\prime}-x y l y l$ crown ethers in nine steps from $p$-cresol utilizing the methoxymethyl group for the protection of the phenolic $\mathrm{OH}$ group. In their synthesis of $2^{\prime}$ - hydroxy $-1^{\prime}, 3^{\prime}-\mathrm{xylyl}$ - 15 - crown - 4 and 18 - crown - 5 (1a and $1 \mathrm{~b}, \mathrm{H}$ for $\mathrm{CH}_{3}$ ) McKervey and Mulholland ${ }^{6}$ protected the $\mathrm{OH}$ group by methylation and after the Williamson ether synthesis the selective cleavage of the methyl aryl ether bond was performed with anhydrous lithium iodide in pyridine at $100^{\circ}$. Under these conditions the benzylic ether bonds were found to be stable.

\section{RESULTS AND DISCUSSION}

To extend the Koenig multistep synthesis to a whole series of crown ethers was obviously not attractive and at the start of our work McKervey and Mulhollands method appeared to be suitable for the synthesis of crown ethers with ringsizes of 24 to 30 atoms. Therefore we prepared the crown ethers $1 \mathrm{~b}-1 \mathrm{e}$ in yields of $27-71 \%$ by the reaction of 2,6 - bis(bromomethyl)anisole with tetra-, penta-, hexa- and heptaethylene glycol. However demethylation of 1c-le with anhydrous lithium iodide in pyridine at $100^{\circ}$ could not be realized under similar conditions to those reported for $1 \mathrm{a}$ and $1 \mathrm{~b} .{ }^{6}$ Other methods that have been used for the cleavage of the methyl aryl ether bond similarly failed. ${ }^{16}$ The different reactivities of the 15 - and 18 -membered rings $1 \mathrm{a}$ and $\mathbf{1 b}$ on one hand and the larger crown ethers lc-le on the other is probably due to the favourable "intramolecular" crown ether catalysis proposed by McKervey and Mulholland ${ }^{6}$ for the smaller rings.

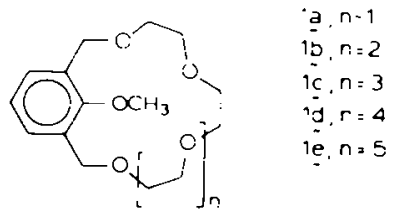

Since complexation of lithium salt usually decreases strongly with increasing ringsize ${ }^{14}$ and since 2,6dimethylanisole itself does not react with lithium iodide, ${ }^{6}$ this crown ether catalysis cannot operate in larger rings. 
Therefore we decided to investigate the use of another protecting group that fulfills the conditions mentioned. The allyl group potentially meets these requirements since allyl ethers are stable in acid and base but readily isomerize on $\mathrm{Pd}-\mathrm{C}$ to give the analogous vinyl ethers. ${ }^{17}$ The latter readily are cleaved under dilute acidic conditions.

The starting material for the synthesis of the crown ethers 8, 4 - chloro - 2,6 - bis(bromomethyl) - 1 - (2 propenyloxy)benzene (5), was prepared in three steps from 4-chlorophenol (Scheme 1). Bishydroxymethylation of 4-chlorophenol according to Openshaw ${ }^{18}$ followed by reaction of the resulting 4 - chloro - 2,6 - bis(hydroxymethyl)phenol (3) with one equivalent of allylbromide gave 4 in an overall yield of $42 \%$. Reaction of 4 with phosphorus tribromide gave the dibromide 5 in a yield of $90 \%$. Reaction of 5 with one equivalent of tetra-, penta-, hexa-. hepta- and octaethylene glycol respectively gave the corresponding crown ethers 6a-e. The yields of the crown ether prepared and the base used are given in Table 1.

In several cases $(n=3,4,5)$ the isolated products were shown to contain the analogous vinyl derivatives 7 , indicating that some base-catalyzed isomerization had taken place. ${ }^{19}$ The ${ }^{1} \mathrm{H}$ NMR spectrum of 6 a showed that the benzylic protons were nonequivalent indicating that the intra-annular allyloxy group at the 2 -position of this 18-membered ring inhibits the conformational changes by which the two faces of the macroring become equivalent on the 'H NMR time scale. This feature has been observed for other $2^{\prime}$-substituted $1^{\prime}, 3^{\prime}$-xylyl crown ethers and there is a clear correlation between the size of substituents, the ringsize $e^{4.6 .15}$ and the rate of conformational changes.
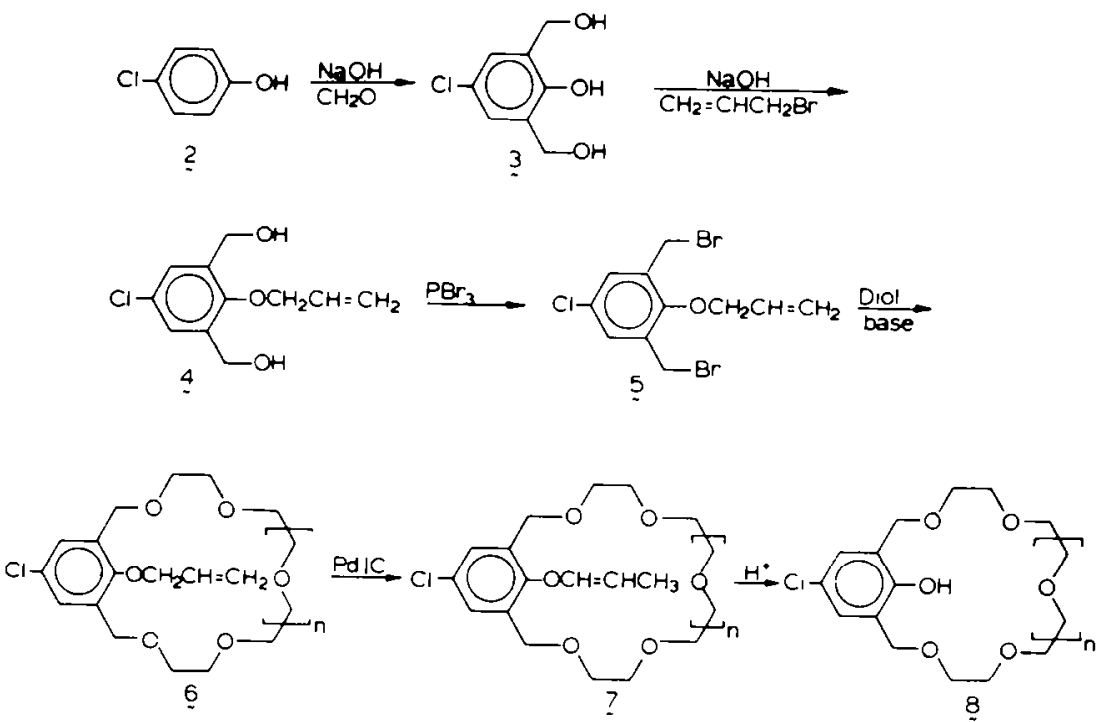

a. $n=1 \quad$ b, $n=2 \quad c, n=3 \quad$ d, $n=4 \quad$ e, $n=5$

Scheme 1.

Table 1. Reactions of 5 with polyethylene glycols

\begin{tabular}{|c|c|c|c|}
\hline C'E & $n$ & BASE & YIELD \\
\hline 6ia & 1 & $\mathrm{NaH}$ & $22^{a}$ \\
\hline ba & 1 & $\mathrm{NaH}$ & $53^{b}$ \\
\hline$\underset{\sim}{6 a}$ & 1 & $\mathrm{KO}: \mathrm{Bu}$ & $30^{\mathrm{b}}$ \\
\hline$\stackrel{6 b}{\sim}$ & 2 & $\mathrm{NaH}$ & 26 \\
\hline $6 c$ & 3 & $\mathrm{KO}: \mathrm{Bu}$ & 52 \\
\hline$\underset{\sim}{\text { od }}$ & 4 & $\mathrm{KO}: \mathrm{Bu}$ & 37 \\
\hline i.e & 5 & $\mathrm{KO}: \mathrm{Bu}$ & 47 \\
\hline
\end{tabular}

a OVERALL, IIELD OF PHENOL 8 a

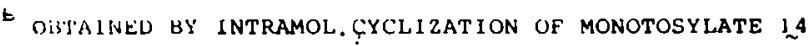

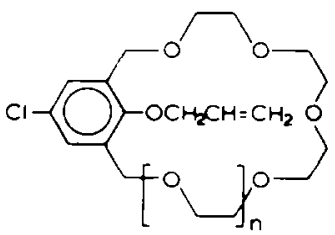


Crown ethers 6 were converted into 7, using the procedure of Boss and Scheffold, ${ }^{17}$ with $\mathrm{Pd}-\mathrm{C}$ in aqueous ethanol and simultaneously the crown ethers 7 formed were hydrolyzed by $p$-toluenesulfonic acid to the $5^{\prime}$. chloro $-2^{\prime}$ - hydroxy $-1^{\prime}, 3^{\prime}-x y l y l$ crown ethers $8 \mathrm{a} e$. The yields varied from 64 to $87 \%$, calculated on 6 . The crown ethers 6 and 8 have been characterized by mass spectrometry, ${ }^{1} \mathrm{H}$ and ${ }^{13} \mathrm{C}$ NMR spectroscopy and by elemental analysis for crystaliine crown ethers.

Crown ether 6a was also obtained by an alternative synthetic route as indicated in Scheme 2 . The intramolecular cyclization reaction of monotosylate 14, that was obtained as a by-product of ditosylate 10 (see Scheme 3 ), was effected by sodium hydride or potassium t-butoxide, giving $6 \mathrm{a}$ in 53 and $30 \%$ yield respectively.

$1^{\prime}, 3^{\prime}$ - Xylyl - dibenzo - 30 - crown - 912 has been prepared in a different way. It comprises a high yield synthesis of a 30-membered crown ether having nine 0 donor atoms in the macroring in addition to an intraannular phenolic group. The synthesis of the macrocycle 12 is outlined in Scheme 3. Dibromide 5 reacted with an excess of diethylene glycol in the presence of base to give in $70 \%$ yield the diol 9, which gave with $p$ toluenesulfonyl chloride a mixture of the ditosylate $\mathbf{1 0}$ (58\%) and the monotosylate $14(9 \%)$. The two compounds were separated by chromatography. Reaction of ditosylate 10 with one equivalent of bis[2 - (o hydroxyphenoxy)ethyl]ether (11) in acetonitrile in the presence of four equivalents of cesium fluoride ${ }^{20}$ gave the crown ether 12 in a yield of $87 \%$. This result demonstrates again the remarkable template effect of the cesium cation in ringclosure reactions. ${ }^{20.21}$
The allyl group in 12 was also removed by reaction with $\mathrm{Pd}-\mathrm{C}$ in the presence of $p$-toluenesulphonic acid to give crown ether 13 , that was readily characterized by the usual spectroscopic techniques. Its ' $\mathrm{H}$ NMR spectrum with separate absorptions for the nonbenzylic ether protons at $\delta 3.70(\mathrm{~s}, 8 \mathrm{H}), \delta 3.88(\mathrm{t}, 8 \mathrm{H}), \delta 4.17(\mathrm{t}, 8 \mathrm{H})$ and an absorption at $\delta 7.81$ for the $\mathrm{OH}$ proton confirmed the structure assignment. The complexation of various neutral molecules with crown ethers 8 and 13 is currently under investigation, and was already demonstrated by the selective complexation of $8 d$ with water. A crystalline 1:1 complex was obtained. Hitherto only two crystalline complexes of a crown ether and water had been reported. ${ }^{13.22}$ An X-ray analysis in order to establish the nature of the binding will be carried out in the near future.

\section{CONCLUSIONS}

The synthesis of $2^{\prime}$ - hydroxy - $1^{\prime}, 3^{\prime}$ - xylyl crown ethers utilizing the allyl group to protect the $\mathrm{OH}$ group during the Williamson ether cyclization reaction has been shown to be a general method.

We found no limitation due to steric hindrance in the ring-closure reaction for ringsizes that vary from 18 - to 30-membered rings. Cleavage of the $\mathrm{C}-\mathrm{O}$ bond in the crown ethers by isomerization of the $\mathrm{C}=\mathrm{C}$ double bond followed by acid-catalyzed hydrolysis of the cis-enol ethers leaves the macrocyclic ring unaffected.

\section{EXPERIMENTAL}

M.ps were recorded on a Reichert m.p. microscope, the ' $H$ NMR-spectra on a Bruker WP 80-FT and the ${ }^{13} \mathrm{C}$ NMR-spectra

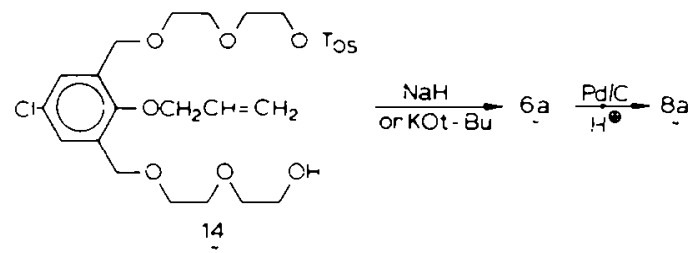

Scheme 2.

5
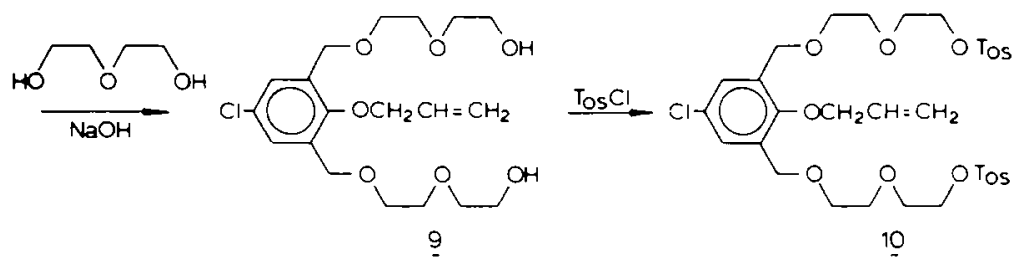

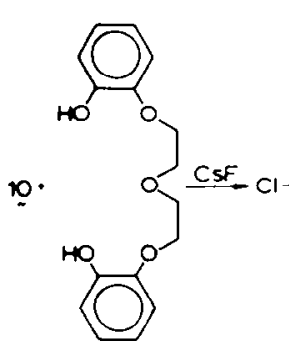

11

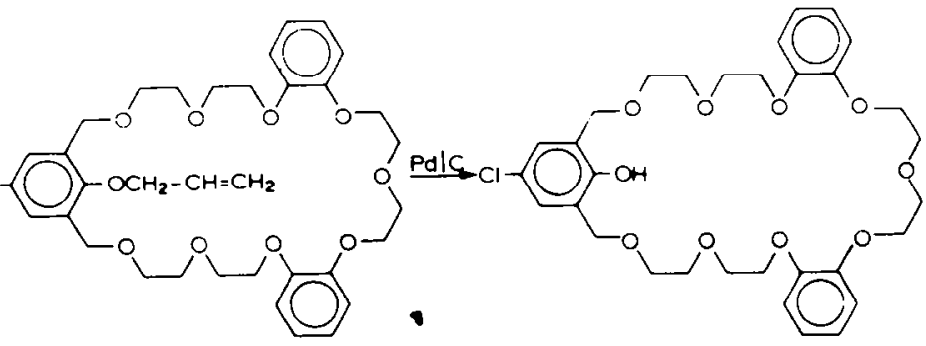

12

Scheme 3. 
on a Varian XL-100-spectrometer in $\mathrm{CDCl}_{3}$ with TMS as internal standard. Mass spectra were obtained with a Varian Mat $311 \mathrm{~A}$ and IR spectra with a Perkin-Elmer 257 spectrometer. Elemental analyses were carried out by the Elemental Analytical Section of the Institute for Organic Chemistry TNO, Utrecht, the Netherlands, under the supervision of W. J. Buis.

Tetraethylene glycol refers to Aldrich reagent. Penta- and heptaethylene glycol were prepared as described by Krespan. ${ }^{23}$ Hexa- and octaethylene glycol were prepared respectively from sodium diethylene glycol and diethylene glycol dichloride (Fluka) and from potassium diethylene glycol and bis[2 - (2 - chloroethoxy)ethyl]ether ${ }^{24}$ by the same procedure with minor modifications. The glycols were distilled under vacuum until glc analysis showed that they were $>95 \%$ pure. 2,6 - Bis(bromomethyl)anisole ${ }^{25}$ and 4 - chloro - 2,6 - bis(hydroxymethyl)phenol $(3)^{18}$ were prepared according to known methods.

4 - Chloro - 2,6 - bis(hydroxymethyl) - 1 - (2 - propenyloxy)benzene (4) and 5 were prepared as follows: $5.2 \mathrm{ml}$ $(60.6 \mathrm{mmol})$ allylbromide was added dropwise to a refluxing soln of $12.5 \mathrm{~g}(60 \mathrm{mmol}) \mathrm{Na}$ salt of $3^{18}$ in $100 \mathrm{ml} \mathrm{MeOH}$. After the addition refluxing was continued for $6 \mathrm{hr}$, the soln was concentrated in vacuo and to the residue water and ether were added. Separation of the organic layer, drying and concentration in vacuo afforded a solid which on crystallization from chloroform, gave $4(8.4 \mathrm{gr}, 62 \%)$, m.p. $109-110^{\circ}$. IR (KBr): 1030, 1060, 1200, 1440,2890 and $3300 \mathrm{~cm}{ }^{1}$. ${ }^{1} \mathrm{H}$ NMR $\left(\mathrm{CDCl}_{3}\right): \delta 4.24(\mathrm{~d}, J=6 \mathrm{~Hz}$, $\mathrm{OH}, 2 \mathrm{H}) ; \delta 4.43\left(\mathrm{~m}, \mathrm{OCH}_{2}, 2 \mathrm{H}\right) ; \delta 4.72\left(\mathrm{~d}, J=6 \mathrm{~Hz}, \mathrm{ArCH}_{2}, 4 \mathrm{H}\right)$; $\delta 5.24\left(\mathrm{~m}\right.$ of d, $\left.J=1.5,10 \mathrm{~Hz},=\mathrm{CH}_{2}, 1 \mathrm{H}\right) ; \delta 5.42(\mathrm{~m}$ of $\mathrm{d}, J=1.5$, $\left.17 \mathrm{~Hz},=\mathrm{CH}_{2}, 1 \mathrm{H}\right) ; \delta 6.14$ (ddt, $J=1.5,10$ and $\left.17 \mathrm{~Hz},=\mathrm{CH}-, 1 \mathrm{H}\right)$; $\delta 7.41$ (s, ArH, 2H). ${ }^{13} \mathrm{C} \mathrm{NMR}\left(\mathrm{CDCl}_{3}\right): \delta 75.6\left(\mathrm{t}, \mathrm{OCH}_{2}\right) ; \delta 117.2$ $\left(\mathrm{t},=\mathrm{CH}_{2}\right) ; \delta 126.1\left(\mathrm{~s}, \mathrm{C}_{4}\right) ; \delta 127.3\left(\mathrm{~d}, \mathrm{C}_{3.5}\right) ; \delta 134.8(\mathrm{~d},=\mathrm{CH}-) ; \delta$ $138.2\left(\mathrm{~s}, \mathrm{C}_{2.6}\right) ; \delta 154.6\left(\mathrm{~s}, \mathrm{C}_{1}\right)$. MS: $\mathrm{M}^{+} 228.055$, Calc. 228.055. (Found: $\mathrm{C}, 57.81 ; \mathrm{H}, 5.62 ; \mathrm{O}, 15.16$. Calc. for $\mathrm{C}_{11} \mathrm{H}_{13} \mathrm{O}_{3} \mathrm{Cl}$ : C. $57.77 ; \mathrm{H}, 5.74 ; \mathrm{O}, 15.50 \%)$.

A soln of $18.0 \mathrm{gr}(66 \mathrm{mmol})$ fresh distilled $\mathrm{PBr}_{3}$ in $75 \mathrm{ml}$ toluene was added dropwise to a suspension of $13.8 \mathrm{gr}(60 \mathrm{mmol})$ diol $4 \mathrm{in}$ $400 \mathrm{ml}$ toluene $/ 75 \mathrm{ml}$ ether at RT. After stirring for $20 \mathrm{hr} 10 \mathrm{~m}$ ] water was added and stirring was continued for $\frac{1}{2} \mathrm{hr}$. The soln was extracted with a sat $\mathrm{NaHCO}_{3} \mathrm{aq}$, washed with water, dried and concentrated in vacuo. The residue was crystallized from hexane to give $5(19.0 \mathrm{gr}, 90 \%)$, m.p. $88-89^{\circ}$. IR (KBr): 1205,1220 , 1240,1255 and $1460 \mathrm{~cm}^{-1}{ }^{1} \mathrm{H}$ NMR $\left(\mathrm{CDCl}_{3}\right): \delta 4.48\left(\mathrm{~s}, \mathrm{ArCH}_{2}\right.$, $4 \mathrm{H}) ; \delta 4.63\left(\mathrm{t}\right.$ of d, $\left.J=1.5,5.5 \mathrm{~Hz}, \mathrm{OCH}_{2}, 2 \mathrm{H}\right) ; \delta 5.34(\mathrm{~m}$ of d, $\left.J=10 \mathrm{~Hz},=\mathrm{CH}_{2}, 1 \mathrm{H}\right) ; \delta 5.51\left(\mathrm{~m}\right.$ of d, $\left.J=17 \mathrm{~Hz},=\mathrm{CH}_{2}, 1 \mathrm{H}\right) ; \delta$ 6.17 (ddt, $J=5.5,10$ and $17 \mathrm{~Hz}$ ); $\delta 7.36$ (s, ArH, 2H). ${ }^{13} \mathrm{C}$ NMR $\left(\mathrm{CDCl}_{3}\right): \delta 75.3\left(\mathrm{t}, \mathrm{OCH}_{2}\right) ; \delta 118.1\left(\mathrm{t},=\mathrm{CH}_{2}\right) ; \delta 129.7\left(\mathrm{~s}, \mathrm{C}_{4}\right) ; \delta$ $131.6\left(\mathrm{~d}, \mathrm{C}_{3,5}\right) ; \delta 132.6(\mathrm{~d},=\mathrm{CH}-) ; \delta 133.7\left(\mathrm{~s}, \mathrm{C}_{2.6}\right) ; \delta 153.7\left(\mathrm{~s}, \mathrm{C}_{1}\right)$. MS: $\mathrm{M}^{+}$351.887, Calc. 351.887. (Found: C, 37.30; $\mathrm{H}, 3.05 ; \mathrm{Br}, \mathrm{Cl}$, 8.55 meq/g. Calc. for $\mathrm{C}_{11} \mathrm{H}_{11} \mathrm{OBr}_{2} \mathrm{Cl}: \mathrm{C}, 37.27 ; \mathrm{H}, 3.13 ; \mathrm{Br}, \mathrm{Cl}$, $8.46 \mathrm{meq} / \mathrm{g}$ ).

General procedure $A$. A soln of $10 \mathrm{mmol} 2,6$ - bis(bromomethyl)anisole and $10 \mathrm{mmol}$ of the appropriate glycol in 100 mmol THF was added dropwise under $\mathrm{N}_{2}$ to a refluxing suspension of $25 \mathrm{mmol} \mathrm{NaH}$ in $150 \mathrm{ml} \mathrm{THF}$. After the addition was complete, refuxing was continued for $30 \mathrm{~min}$. The mixture was allowed to cool to RT and stirred for $16 \mathrm{hr}$. The salt was removed by filtration and the solution was concentrated in vacuo.

General procedure $B$. A soln of $10 \mathrm{mmol}$ of the appropriate glycol in $50 \mathrm{ml}$ THF was added dropwise under $\mathrm{N}_{2}$ to a suspension of $22 \mathrm{mmol} \mathrm{NaH}$ in $50 \mathrm{ml}$ THF. After refluxing for $\frac{1}{2} \mathrm{hr}$ a soln of $10 \mathrm{mmol}$ of 5 in $50 \mathrm{ml}$ THF was added dropwise and the resulting mixture was heated at $60^{\circ}$ for $2 \mathrm{hr}$. After cooling the mixture was filtered and the soln was concentrated in vacuo.

$2^{\prime}$ - Methoxy - 1',3' - xy/yl - 18 - crown - 5 lb was prepared according to procedure $A$. Purification was accomplished by distillation $\left(200-240^{\circ} \mathrm{C}, 3 \times 10^{-2} \mathrm{~mm}\right)$ and crystallization from hexane-ether; yield $69.5 \%$ (lit $^{6} 58 \%$ ), m.p. $45-47^{\circ}$. IR (KBr): 775 , $790,1105,1470,1600$ and $2870 \mathrm{~cm}^{-1}$. 'H NMR $\left(\mathrm{CDCl}_{3}\right): \delta 3.48$, $3.60\left(2 \mathrm{~s}, \mathrm{OCH}_{2} \mathrm{CH}_{2} \mathrm{O}, 16 \mathrm{H}\right) ; \delta 4.14\left(\mathrm{~s}, \mathrm{OCH}_{3}, 3 \mathrm{H}\right) ; \delta 4.60(\mathrm{~s}$, $\left.\mathrm{ArCH}_{2}, 4 \mathrm{H}\right) ; \delta 6.90-7.40$ (m, ArH, 3H). ${ }^{13} \mathrm{C} \mathrm{NMR}\left(\mathrm{CDCl}_{3}\right): \delta 64.9$ (q. $\left.\mathrm{OCH}_{3}\right) ; \delta 122.8$ (s, $\left.\mathrm{C}_{\mathrm{s}}\right) ; \delta 131.7\left(\mathrm{~s}, \mathrm{C}_{1,3}\right) ; \delta 131.9\left(\mathrm{~d}, \mathrm{C}_{4,6}\right) ; \delta$ 159.4 (s, C2). MS: $\mathrm{M}^{-}$326.173, Calc. 326.173. (Found: $\mathrm{C}, 62.59 ; \mathrm{H}$, 8.17. Calc. for $\mathrm{C}_{17} \mathrm{H}_{26} \mathrm{O}_{6}: \mathrm{C}, 62.55 ; \mathrm{H}, 8.04 \%$ ).

$2^{\prime}$ - Methoxy - 1',3'-xylyl - 21 - crown - 6 lc was prepared according to procedure $A$. The residue was dissolved in ether and the organic layer washed with water, dried and concentrated in vacuo. Purification of the solid material was accomplished by elution with $\mathrm{MeOH}$; yield $27 \%$, m.p. $82.5-83.5^{\circ}$. IR (KBr): 770 , $790,1100,1465,1600$ and $2870 \mathrm{~cm}^{-1}$. ' $\mathrm{H} \mathrm{NMR}\left(\mathrm{CDCl}_{3}\right): \delta 3.53$, 3.56 and $3.67\left(3 \mathrm{~s}, \mathrm{OCH}_{2} \mathrm{CH}_{2} \mathrm{O}, 2 \mathrm{OH}\right) ; \delta 4.01\left(\mathrm{~s}, \mathrm{OCH}_{3}, 3 \mathrm{H}\right) ; \delta 4.58$ (s, $\left.\mathrm{ArCH}_{2}, 4 \mathrm{H}\right) ; \delta 6.90-7.40(\mathrm{~m}, \mathrm{ArH}, 3 \mathrm{H}) .{ }^{13} \mathrm{C} \mathrm{NMR}\left(\mathrm{CDCl}_{3}\right): \delta$ $64.3\left(\mathrm{q}, \mathrm{OCH}_{3}\right) ; \delta 123.5\left(\mathrm{~s}, \mathrm{C}_{5}\right) ; \delta 131.4\left(\mathrm{~s}, \mathrm{C}_{1,3}\right) ; \delta 131.4\left(\mathrm{~d}, \mathrm{C}_{4,6}\right)$; $\delta 158.6$ (s, $\mathrm{C}_{2}$ ). MS: $\mathrm{M}^{+}$370.199, Calc. 370.199. (Found: C, 61.43; $\mathrm{H}, 8.02 ; \mathrm{O}, 30.55$. Calc. for $\mathrm{C}_{19} \mathrm{H}_{30} \mathrm{O}_{7}: \mathrm{C}, 61.59 ; \mathrm{H}, 8.18 ; \mathrm{O}$, $30.23 \%)$.

$2^{\prime}-$ Methoxy - 1',3'-xylyl - 24 - crown - 7 1d was prepared according to procedure $A$, with the modification that $\mathrm{KO} t-\mathrm{Bu}$ was used as the base. The residue was purified by chromatography (ether-THF 9/1), yield $71 \%$ (referred to used dibromide 5). The oily compound crystallized after standing, m.p. $47-48^{\circ}$. IR (KBr): $775,790,1105,1465,1600$ and $2870 \mathrm{~cm}^{-1}$. ${ }^{1} \mathrm{H}$ NMR $\left(\mathrm{CDCl}_{3}\right): \delta 3.57,3.59$ and $3.68\left(3 \mathrm{~s}, \mathrm{OCH}_{2} \mathrm{CH}_{2} \mathrm{O}, 24 \mathrm{H}\right) ; \delta 3.92(\mathrm{~s}$, $\left.\mathrm{OCH}_{3}, 3 \mathrm{H}\right) ; \delta 4.62\left(\mathrm{~s}, \mathrm{ArCH}_{2}, 4 \mathrm{H}\right) ; \delta 7.00-7.50(\mathrm{~m}, \mathrm{ArH}, 3 \mathrm{H}) .{ }^{13} \mathrm{C}$ NMR $\left(\mathrm{CDCl}_{3}\right): \delta 63.3\left(\mathrm{q}, \mathrm{OCH}_{3}\right) ; \delta 123.7\left(\mathrm{~s}, \mathrm{C}_{5}\right) ; \delta 130.5\left(\mathrm{~d}, \mathrm{C}_{4.6}\right)$; $\delta 131.4\left(\mathrm{~s}, \mathrm{C}_{1.3}\right) ; \delta 157.5\left(\mathrm{~s}, \mathrm{C}_{2}\right)$. MS: $\mathrm{M}^{+}$414.225, Calc. 414.225. (Found: $\mathrm{C}, 60.99 ; \mathrm{H}, 8.17 ; \mathrm{O}, 30.84$. Calc. for $\mathrm{C}_{21} \mathrm{H}_{34} \mathrm{O}_{8}: \mathrm{C}, 60.84$; $\mathrm{H}, 8.28 ; 0,30.88 \%)$.

$2^{\prime}$ - Methoxy - 1',3' - xy/y/ - 27 - crown - 8 le was prepared according to procedure $A$, with the modification that $\mathrm{KO} t-\mathrm{Bu}$ was used as the base. The residue was purified by chromatography on silicagel $\left(\mathrm{CHCl}_{3}\right)$ and distillation (b.p. 200-225 , $10^{-2} \mathrm{~mm}$ ), yield $30 \%$. IR (neat): $770,790,1100,1465,1600$ and $2870 \mathrm{~cm}^{-1}$. ' $\mathrm{H}$ NMR $\left(\mathrm{CDCl}_{3}\right): \delta 3.50-3.75\left(\mathrm{~m}, \mathrm{OCH}_{2} \mathrm{CH}_{2} \mathrm{O}, 28 \mathrm{H}\right)$; $\delta 3.87\left(\mathrm{~s}, \mathrm{OCH}_{3}, 3 \mathrm{H}\right) ; \delta 4.63\left(\mathrm{~s}, \mathrm{ArCH}_{2}, 4 \mathrm{H}\right) ; \delta 7.00-7.50(\mathrm{~m}$, $\mathrm{ArH}, 3 \mathrm{H}) .{ }^{13} \mathrm{C} \mathrm{NMR}\left(\mathrm{CDCl}_{3}\right) ; \delta 62.8\left(\mathrm{q}, \mathrm{OCH}_{3}\right) ; \delta 123.8(\mathrm{~s}, \mathrm{Cs}) ; \delta$ $129.9\left(\mathrm{~d}, \mathrm{C}_{4.6}\right) ; \delta 131.3\left(\mathrm{~s}, \mathrm{C}_{1,3}\right) ; \delta 157.0\left(\mathrm{~s}, \mathrm{C}_{2}\right)$. MS: $\mathrm{M}^{+} 458.248$, Calc. 458.252. (Found: $\mathrm{C}, 59.39 ; \mathrm{H}, 8.23$. Calc. for $\mathrm{C}_{23} \mathrm{H}_{38} \mathrm{O}_{9}: \mathrm{C}$, $60.24 ; \mathrm{H}, 8.35 \%)$.

$5^{\prime}$ - Chloro - 2' - (2 - propenyloxy) - 1',3' - xylyl - 18 - crown - 5 6a was prepared according to procedure B. An analytical sample was obtained upon chromatography on silicagel $\left(\mathrm{CHCl}_{3}-\mathrm{EtOAc}\right.$ 6/4) and crystallization from $\mathrm{CHCl}_{3}-\mathrm{PE} 60-80$ at low temp, m.p. $96-97^{\circ}$. IR (KBr): $1100,1245,1350,1450$ and $2880 \mathrm{~cm}^{-1}$. ${ }^{1} \mathrm{H}$ NMR $\left(\mathrm{CDCl}_{3}\right): \delta 3.28-3.73\left(\mathrm{~m}, \mathrm{OCH}_{2} \mathrm{CH}_{2} \mathrm{O}, 16 \mathrm{H}\right) ; \delta 4.13$ (d. $\left.J=10.5 \mathrm{~Hz}, \mathrm{ArCH}_{2}, 2 \mathrm{H}\right) ; \delta 4.53+4.75-4.92\left(\mathrm{~m}, \mathrm{OCH}_{2}, 2 \mathrm{H}\right) ; \delta$ 4.92 (d, $J=10.5 \mathrm{~Hz}, \mathrm{ArCH}_{2}, 2 \mathrm{H}$ ); $\delta 5.25$ (m of d, $J=10 \mathrm{~Hz}$, $\left.=\mathrm{CH}_{2}, 1 \mathrm{H}\right) ; \delta 5.50\left(\mathrm{~m}\right.$ of $\left.\mathrm{d}, J=16 \mathrm{~Hz},=\mathrm{CH}_{2}, 1 \mathrm{H}\right) ; \delta 6.21$ (ddt, $J=5,10$ and $16 \mathrm{~Hz},=\mathrm{CH}-, 1 \mathrm{H}) ; \delta 7.26,7.27(2 \mathrm{~s}, \mathrm{ArH}, 2 \mathrm{H}) \cdot{ }^{13} \mathrm{C}$ NMR $\left(\mathrm{CDCl}_{3}\right): \delta 77.3\left(\mathrm{t}, \mathrm{OCH}_{2}\right) ; \delta 115.4\left(\mathrm{t},=\mathrm{CH}_{2}\right) ; \delta 131.3(\mathrm{~d}$, $\left.\mathrm{C}_{4.6}\right) ; \delta 133.3\left(\mathrm{~s}, \mathrm{C}_{5}\right) ; \delta 133.8\left(\mathrm{~s}, \mathrm{C}_{1,3}\right) ; \delta 135.7(\mathrm{~d},=\mathrm{CH}-) ; \delta 156.9$ (s, $\mathrm{C}_{2}$ ). MS: $\mathrm{M}^{+} 386.150$, Calc. 386.150. (Found: C, 59.07; H, 7.06. Calc. for $\mathrm{C}_{19} \mathrm{H}_{27} \mathrm{O}_{6} \mathrm{Cl}$ : C, 58.99; $\mathrm{H}, 7.03 \%$ ).

$5^{\prime}$ - Chloro - 2' - (2 - propenyloxy) - $1^{\prime}, 3^{\prime}-x y / y l-21-$ crown - 6 6b was prepared according to procedure B. Purification was accomplished by chromatography on silicagel (EtOAc) and crystallization from heptane; yield $26 \%$, m.p. 46-47 . IR (KBr): 1035 , $1100,1210,1250,1355,1455$ and $2870 \mathrm{~cm}^{-1}$. 'H NMR $\left(\mathrm{CDCl}_{3}\right): \delta$ $3.53,3.55$ and $3.64\left(3 \mathrm{~s}, \mathrm{OCH}_{2} \mathrm{CH}_{2} \mathrm{O}, 20 \mathrm{H}\right) ; \delta 4.53$ (s, $\mathrm{ArCH}_{2}, 4 \mathrm{H}$ ); $\delta 4.66$ ( $\mathrm{t}$ of $\mathrm{d}, J=1.5$ and $5 \mathrm{~Hz}, \mathrm{OCH}_{2}, 2 \mathrm{H}$ ); $\delta 5.30$ (m of d, $\left.J=10 \mathrm{~Hz},=\mathrm{CH}_{2}, 1 \mathrm{H}\right) ; \delta 5.54\left(\mathrm{~m}\right.$ of $\left.\mathrm{d}, J=17 \mathrm{~Hz},=\mathrm{CH}_{2}, 1 \mathrm{H}\right) ; \delta$ 6.23 (ddt, $J=5,10$ and $17 \mathrm{~Hz},=\mathrm{CH}-, 1 \mathrm{H}) ; \delta 7.32$ (s, ArH, 2H). ${ }^{13} \mathrm{C} \mathrm{NMR}\left(\mathrm{CDCl}_{3}\right): \delta 76.9\left(\mathrm{t}, \mathrm{OCH}_{2}\right) ; \delta 116.2\left(\mathrm{t},=\mathrm{CH}_{2}\right) ; \delta 128.4(\mathrm{~s}$, $\left.\mathrm{C}_{5}\right) ; \delta 130.6\left(\mathrm{~d}, \mathrm{C}_{4.6}\right) ; \delta 133.5\left(\mathrm{~s}, \mathrm{C}_{1.3}\right) ; \delta 134.6(\mathrm{~d},=\mathrm{CH}-) ; \delta 155.6$ (s, $\mathrm{C}_{2}$ ). MS: $\mathrm{M}^{+}$430.176, Calc. 430.176. (Found: C, 58.82; H, 7.14; $\mathrm{Cl}, 8.29 ; \mathrm{O}, 25.75$. Calc. for $\mathrm{C}_{21} \mathrm{H}_{31} \mathrm{O}_{7} \mathrm{Cl}: \mathrm{C}, 58.52 ; \mathrm{H}, 7.27 ; \mathrm{Cl}$, $8.23 ; 0,25.99 \%)$.

$5^{\prime}$ - Chloro - 2' - (2 - propenyloxy) - 1'.3'-xylyl - 24-crown - 7 6c was prepared according to procedure $\mathrm{B}$, however $\mathrm{KO} t-\mathrm{Bu}$ was used as the base. Purification was accomplished by chromatography on silicagel (EtOAc). From spectral data it appeared that a 1:1 mixture of the allyl and vinylether was present, yield $52 \%$. IR (neat): $1110,1210,1250,1355,1450$ and $2880 \mathrm{~cm}^{-1}$. ${ }^{1} \mathrm{H}$ NMR $\left(\mathrm{CDCl}_{3}\right.$ ): $\delta 1.77$ (dd, $J=2$ and $7 \mathrm{~Hz},=\mathrm{CH}-\mathrm{CH}_{3}, 1.5 \mathrm{H}$ ); $\delta$ $3.55,3.60,3.68\left(3 \mathrm{~s}, \mathrm{OCH}_{2} \mathrm{CH}_{2} \mathrm{O}, 24 \mathrm{H}\right.$ ); $\delta 4.50$ (t of $\mathrm{d}, J=1.2$ and $\left.5 \mathrm{~Hz}, \mathrm{OCH}_{2}, \mathrm{IH}\right) ; \delta 4.58\left(\mathrm{~s}, \mathrm{ArCH}_{2}, 4 \mathrm{H}\right) ; \delta 5.28$ (m of d, $\left.J=10 \mathrm{~Hz},=\mathrm{CH}_{2}, 0.5 \mathrm{H}\right) ; \delta 5.46$ (m of d, $\left.J=17 \mathrm{~Hz},=\mathrm{CH}_{2}, 0.5 \mathrm{H}\right)$; $\delta 5.90-6.40(\mathrm{~m},=\mathrm{CH}-, 1.5 \mathrm{H}) ; \delta 7.37,7.42(2 \mathrm{~s}, \mathrm{ArH}, 2 \mathrm{H}),{ }^{13} \mathrm{C}$ NMR $\left(\mathrm{CDCl}_{3}\right) ; \delta 76.1\left(\mathrm{t}, \mathrm{OCH}_{2}\right) ; \delta 102.8(\mathrm{~d}, \mathrm{OCH}=) ; \delta 116.8(\mathrm{t}$, 
$\left.=\mathrm{CH}_{2}\right) ; \delta 128.8\left(\mathrm{~d}, \mathrm{C}_{46}\right) ; \delta 128.8\left(\mathrm{~s}, \mathrm{C}_{5}\right) ; \delta 129.7\left(\mathrm{~d}, \mathrm{C}_{4,6}\right) ; \delta 129.7$ $\left(\mathrm{s}, \mathrm{C}_{3}\right) ; \delta 133.0\left(\mathrm{~s}, \mathrm{C}_{1.3}\right) ; \delta 133.5\left(\mathrm{~s}, \mathrm{C}_{1.3}\right) ; \delta 133.9(\mathrm{~d},=\mathrm{CH}-) ; \delta$ $145.1\left(\mathrm{~d}_{1}=\mathrm{CH}-\right) ; \delta 151.7\left(\mathrm{~s}, \mathrm{C}_{2}\right) ; \delta 154.4\left(\mathrm{~s}, \mathrm{C}_{2}\right) . \mathrm{MS}: \mathrm{M}^{+} 474.201$, Calc. 474.202 .

$5^{\prime}$ - Chloro - 2' - (2 - propenyloxy) - $1^{\prime}, 3^{\prime}-x y l y l-27$ - crown - 8 6d was prepared according to procedure $\mathrm{B}$, however $\mathrm{KO} t-\mathrm{Bu}$ or $\mathrm{KH}$ were used as the base. Purification was accomplished by chromatography on silicagel $\left(\mathrm{CHCl}_{3}-\mathrm{MeOH}, 7 \%\right)$, yield $37 \%$. From spectral data it appeared that about $5 \%$ of vinylether 7d was present. IR (neat): $1110,1200,1350,1450$ and $2860 \mathrm{~cm}^{-1}$. ' $\mathrm{H}$ NMR $\left(\mathrm{CDCl}_{3}\right): \delta 3.62,3.64,3.69\left(3 \mathrm{~s}, \mathrm{OCH}_{2} \mathrm{CH}_{2} \mathrm{O}, 28 \mathrm{H}\right) ; \delta 4.41$ (m of d, $\left.J=5 \mathrm{~Hz}, \mathrm{OCH}_{2}, 2 \mathrm{H}\right) ; \delta 4.54\left(\mathrm{~s}, \mathrm{ArCH}_{2}, 4 \mathrm{H}\right) ; \delta 5.20(\mathrm{~m}$ of d, $\left.J=10 \mathrm{~Hz},=\mathrm{CH}_{2}, 1 \mathrm{H}\right) ; \delta 5.38\left(\mathrm{~m}\right.$ of $\mathrm{d}, J=14 \mathrm{~Hz},=\mathrm{CH}_{2}$, IH); $\delta 6.08$ (ddt, $J=5,10$ and $14 \mathrm{~Hz},=\mathrm{CH}-, 1 \mathrm{H}) ; \delta 7.30$ (s, ArH, $2 \mathrm{H}) .{ }^{3} \mathrm{C}$ NMR $\left(\mathrm{CDCl}_{3}\right): \delta 75.9\left(\mathrm{t}, \mathrm{OCH}_{2}\right) ; \delta 117.2\left(\mathrm{t},=\mathrm{CH}_{2}\right) ; \delta$ $128.4\left(\mathrm{~s}, \mathrm{C}_{5}\right) ; \delta 129.2\left(\mathrm{~d}, \mathrm{C}_{4.6}\right) ; \delta 133.5\left(\mathrm{~s}, \mathrm{C}_{1,3}\right) ; \delta 133.6(\mathrm{~d},=\mathrm{CH}-)$; $\delta 153.8\left(\mathrm{~s}, \mathrm{C}_{2}\right)$. MS: $\mathrm{M}^{+}$518.233, Calc. 518.228.

$5^{\prime}-$ Chloro - 2' - (2 - propenyloxy) - 1',3' - xylyl - 30 - crown - 9 the was prepared according to procedure $\mathrm{B}$, however $\mathrm{KO} \mathrm{t}-\mathrm{Bu}$ was used as the base. Purification was accomplished by chromatography on silicagel $\left(\mathrm{CHCl}_{3}-\mathrm{MeOH}, 7 \%\right)$, yield $47 \%$. IR (neat): $1110,1200,1250,1350,1450$ and $2870 \mathrm{~cm}^{-1}$. ${ }^{1} \mathrm{H}$ NMR $\left(\mathrm{CDCl} \mathrm{l}_{3}\right): \delta$ $3.64,3.65,3.66,3.67$ and $3.70\left(5 \mathrm{~s}, \mathrm{OCH}_{2} \mathrm{CH}_{2} \mathrm{O}, 32 \mathrm{H}\right.$ ); $\delta 4.36$ ( $\mathrm{m}$ of d, $\left.J=5 \mathrm{~Hz}, \mathrm{OCH}_{2}, 2 \mathrm{H}\right) ; \delta 4.56\left(\mathrm{~s}, \mathrm{ArCH}_{2}, 4 \mathrm{H}\right) ; \delta 5.22$ (m of d, $\left.J=10 \mathrm{~Hz},=\mathrm{CH}_{2}, 1 \mathrm{H}\right) ; \delta 5.40\left(\mathrm{~m}\right.$ of $\left.\mathrm{d}, J=14 \mathrm{~Hz},=\mathrm{CH}_{2}, 1 \mathrm{H}\right) ; \delta$ $6.06(\mathrm{ddt}, J=5,10$ and $14 \mathrm{~Hz}) ; \delta 7.35(\mathrm{~s}, \mathrm{ArH}, 2 \mathrm{H}),{ }^{13} \mathrm{C}$ NMR $(\mathrm{CDCl}): \delta 75.7\left(\mathrm{t}, \mathrm{OCH}_{2}\right) ; \delta 117.4\left(\mathrm{t},=\mathrm{CH}_{2}\right) ; \delta 128.6\left(\mathrm{~d}, \mathrm{C}_{4,6}\right) ; \delta$ $129.3\left(\mathrm{~s}, \mathrm{C}_{5}\right) ; \delta 133.3\left(\mathrm{~s}, \mathrm{C}_{1,3}\right) ; \delta 133.3(\mathrm{~d},=\mathrm{CH}) ; \delta 153.3\left(\mathrm{~s}, \mathrm{C}_{2}\right)$. MS: $\mathrm{M}^{+} 562.250$. Calc. 562.245 .

5' - Chloro - 2' - hydroxy - 1',3'-xylyl-18-crown - 58a was prepared from crude 6a according to the procedure of Boss and Scheffold ${ }^{17}$ with the difference that the reaction was carried out in EtOH. Purification was accomplished by chromatography on silicagel ( $\mathrm{CHCl}-\mathrm{EtOAC} 14)$, yield $22 \%$ (referred to dibromide 5), m.p. $62-65^{\circ}$. IR (KBr): $1100,1250,1355,1470,2870$ and $3350 \mathrm{~cm}^{-1}$. 'H NMR ( $\left.\mathrm{CDCl}_{3}\right): \delta 3.68,3.70\left(2 \mathrm{~s}, \mathrm{OCH}_{2} \mathrm{CH}_{2} \mathrm{O}, 16 \mathrm{H}\right)$; $\delta 4.64\left(\mathrm{~s}, \mathrm{ArCH}_{2}, 4 \mathrm{H}\right) ; \delta 7.10(\mathrm{~s}, \mathrm{ArH}, 2 \mathrm{H}) ; \delta 8.0(\mathrm{br} \mathrm{s}, \mathrm{OH}, 1 \mathrm{H})$. ${ }^{13} \mathrm{C}$ NMR $\left(\mathrm{CDCl}_{3}\right) ; \delta 123.6\left(\mathrm{~s}, \mathrm{C}_{5}\right) ; \delta 126.3\left(\mathrm{~s}, \mathrm{C}_{1,3}\right) ; \delta 128.8(\mathrm{~d}$, $\left.\mathrm{C}_{4.6}\right) ; \delta 154.0\left(\mathrm{~s}, \mathrm{C}_{2}\right.$ ). MS: $\mathrm{M}^{+}$346.119. Calc. 346.118. (Found: $\mathrm{C}$, 55.49; $\mathrm{H}, 6.73$. Calc. for $\mathrm{C}_{16} \mathrm{H}_{23} \mathrm{O}_{6} \mathrm{Cl}$ : C, $55.41 ; \mathrm{H}, 6.68 \%$ ).

$5^{\prime}$ - Chloro - 2' - hydroxy - $1^{\prime}, 3^{\prime}-x y l y l-21-c r o w n-68 \mathrm{~b}$ was prepared from $6 \mathrm{~b}$ according to the procedure of Boss and Scheffold ${ }^{17}$ with the difference that the reaction was carried out in EtOH. Purification was accomplished by chromatography on silicagel $\left(\mathrm{CHCl}_{3}-\mathrm{MeOH}, 6 \%\right)$, yield $70 \%$. IR (neai): 1100,1250 1350, 1460, 2860 and $3340 \mathrm{~cm}^{-1}$. ' $\mathrm{H}$ NMR $\left(\mathrm{CDCl}_{3}\right): \delta 3.60,3.68$ and $3.70\left(3 \mathrm{~s}, \mathrm{OCH}_{2} \mathrm{CH}_{2} \mathrm{O}, 2 \mathrm{H}\right) ; \delta 4.66\left(\mathrm{~s}, \mathrm{ArCH}_{2}, 4 \mathrm{H}\right) ; \delta 7.11$ (s, $\mathrm{ArH}, 2 \mathrm{H}) ; \delta 7.89$ (br s, OH, $1 \mathrm{H}) .{ }^{13} \mathrm{C} \mathrm{NMR}\left(\mathrm{CDCl}_{3}\right): \delta 123.6(\mathrm{~s}$, $\left.\mathrm{C}_{5}\right) ; \delta 126.1\left(\mathrm{~s}, \mathrm{C}_{1.3}\right) ; \delta 127.9$ (d. $\left.\mathrm{C}_{4.6}\right) ; \delta 153.1\left(\mathrm{~s}, \mathrm{C}_{2}\right)$. MS: $\mathrm{M}^{*}$ 390.145, Calc. 390.145 .

5' - Chloro - 2' - hydroxy - 1', $3^{\prime}-x y l y l-24-$ crown - 7 8c was prepared from oc according to the procedure of Boss and Scheffold ${ }^{17}$ with the difference that the reaction was carried out in EtOH. Purification was accomplished by chromatography on silicagel $\left(\mathrm{CHCl}_{3}-\mathrm{MeOH}, 7 \%\right)$, yield $64 \%$. IR (neat): $1100,1252$. 1352, 1465,2870 and $3350 \mathrm{~cm}^{-1}$. ' $\mathrm{H}$ NMR $\left(\mathrm{CDCl}_{3}\right): \delta 3.67,3.69$ and $3.72\left(3 \mathrm{~s}, \mathrm{OCH}_{2} \mathrm{CH}_{2} \mathrm{O}, 24 \mathrm{H}\right) ; \delta 4.66\left(\mathrm{~s}, \mathrm{ArCH}_{2}, 4 \mathrm{H}\right) ; \delta 7.13$ (s, $\mathrm{ArH}, 2 \mathrm{H}) ; \delta 7.96(\mathrm{br} \mathrm{s}, \mathrm{OH}, 1 \mathrm{H}),{ }^{13} \mathrm{C}$ NMR $\left(\mathrm{CDCl}_{3}\right): \delta 123.9(\mathrm{~s}$, $\left.\mathrm{C}_{5}\right) ; \delta 126.0\left(\mathrm{~s}, \mathrm{C}_{1.3}\right) ; \delta 127.8\left(\mathrm{~d}, \mathrm{C}_{4,6}\right) ; \delta 152.6\left(\mathrm{~s}, \mathrm{C}_{2}\right)$. MS: $\mathrm{M}^{+}$ 434.170, Calc. 434.171

$5^{\prime}$ - Chloro - 2' - hydroxy - $1^{\prime}, 3^{\prime}-x y l y l-27$ - crown - 8 8d was prepared from 6d according to the procedure of Boss and Scheffold ${ }^{17}$ with the modification that the reaction was carried out in EtOH. Purification was accomplished by chromatography on silicagel $\left(\mathrm{CHCl}_{3}-\mathrm{MeOH}, 7 \%\right)$ and crystallization from wet ether-hexane; yield $87 \%$, m.p. $55^{\circ}$. IR (KBr): $1100,1260,1350$, 1460,2880 and $3440 \mathrm{~cm}^{-1}$. ${ }^{1} \mathrm{H}$ NMR $\left(\mathrm{CDCl}_{3}\right): \delta 2.89$ (br s, $\mathrm{H}_{3} \mathrm{O}^{+}$, $3 \mathrm{H}$ ); $\delta 3.62,3.65,3.67$ and $3.72\left(4 \mathrm{~s}, \mathrm{OCH}_{2} \mathrm{CH}_{2} \mathrm{O}, 28 \mathrm{H}\right.$ ); $\delta 4.65$ (s, $\left.\mathrm{ArCH}_{2}, 4 \mathrm{H}\right) ; \delta 7.14(\mathrm{~s}, \mathrm{ArH}, 2 \mathrm{H}) .{ }^{13} \mathrm{C} \mathrm{NMR}\left(\mathrm{CDCl}_{3}\right): \delta 124.0(\mathrm{~s}$, $\left.\mathrm{C}_{5}\right) ; \delta 126.0\left(\mathrm{~s}, \mathrm{C}_{1,3}\right) ; \delta 128.0\left(\mathrm{~d}, \mathrm{C}_{4.6}\right) ; \delta 152.6\left(\mathrm{~s}, \mathrm{C}_{2}\right) . \mathrm{MS}: \mathrm{M}^{+}$ 478.197, Calc. 478.197. (Found: C, 52.86; H, 7.30; Cl, 7.21. Calc. for $\mathrm{C}_{22} \mathrm{H}_{35} \mathrm{O}, \mathrm{Cl} \cdot \mathrm{H}_{2} \mathrm{O}: \mathrm{C}, 53.16 ; \mathrm{H}, 7.50 ; \mathrm{Cl}, 7.13 \%$ ).

$5^{\prime}$ - Chloro - 2' - hydroxy - 1', $3^{\prime}-x y l y l-30-$ crown - 9 se was prepared from be according to the procedure of Boss and
Scheffold ${ }^{17}$ with the difference that the reaction was carried out in $\mathrm{EtOH}$. Purification was accomplished by chromatography on silicagel $\left(\mathrm{CHCl}_{3}-\mathrm{MeOH}, 7 \%\right)$, yield $65 \%$. IR (KBr): 1100 , 1250 , $1350,1460,2870$ and $3340 \mathrm{~cm}^{-1}$. ${ }^{1} \mathrm{H}$ NMR $\left(\mathrm{CDCl}_{3}\right): \delta 3.65,3.70$ $\left(2 \mathrm{~s}, \mathrm{OCH}_{2} \mathrm{CH}_{2} \mathrm{O}, 32 \mathrm{H}\right) ; \delta 4.63\left(\mathrm{~s}, \mathrm{ArCH}_{2}, 4 \mathrm{H}\right) ; \delta 7.14(\mathrm{~s}, \mathrm{ArH}$, $2 \mathrm{H}) ; \delta 7.37$ (br s, OH, lH). $\left.{ }^{13} \mathrm{C} \mathrm{NMR} \mathrm{(CDCl}\right): \delta 124.0\left(\mathrm{~s}, \mathrm{C}_{5}\right) ; \delta$ $125.6\left(\mathrm{~s}, \mathrm{C}_{1,3}\right) ; \delta 127.3\left(\mathrm{~d}, \mathrm{C}_{4.6}\right) ; \delta 152.5\left(\mathrm{~s}, \mathrm{C}_{2}\right)$. MS: $\mathrm{M}^{+}$522.227, Calc. $\$ 22.223$.

4- Chloro - 2,6 - bis (hydroxyethoxyethoxymethyl) - 1 - (2 propenyloxy)benzene (9). A suspension of $7.1 \mathrm{gr}(20 \mathrm{mmol})$ of 5 and $1.0 \mathrm{gr}(25 \mathrm{mmol}) \mathrm{NaOH}$ in $30 \mathrm{ml}$ diethylene glycol was kept at $110^{\circ}$ for $2 \mathrm{hr}$. To the clear soln water and ether were added. The organic layer was separated and the water layer extracted with $\mathrm{CHCl}_{3}$. The combined organic layers were dried and concentrated in vacuo, yielding almost pure $9(5.6 \mathrm{gr}, 70 \%)$. An analytical sample was obtained by chromatography on silicage ( $\mathrm{CHCl}_{3}-\mathrm{MeOH} 5 \%$ ). IR (neat): $1100,1200,1350,1450,2860$ and $3420 \mathrm{~cm}^{-1}$. ${ }^{1} \mathrm{H} \mathrm{NMR}\left(\mathrm{CDCl}_{3}\right): \delta 3.04$ (br s, OH, 2H); $\delta 3.60,3.62$ and $3.68\left(3 \mathrm{~s}, \mathrm{OCH}_{2} \mathrm{CH}_{2} \mathrm{O}, 16 \mathrm{H}\right) ; \delta 4.37$ (t of d, $\mathrm{J}=5 \mathrm{~Hz}, \mathrm{OCH}_{2}$, $2 \mathrm{H}) ; \delta 4.57\left(\mathrm{~s}, \mathrm{ArCH}_{2}, 4 \mathrm{H}\right) ; \delta 5.26\left(\mathrm{~m}\right.$ of d, $\left.J=10 \mathrm{~Hz},=\mathrm{CH}_{2}, \mathrm{lH}\right)$; $\delta 5.41$ (m of d, $J=16 \mathrm{~Hz},=\mathrm{CH}_{2}, 1 \mathrm{H}$ ); $\delta 6.08$ (ddt, $J=5,10$ and $16 \mathrm{~Hz},=\mathrm{CH}-, 1 \mathrm{H}) ; \delta 7.37(\mathrm{~s}, \mathrm{ArH}, 2 \mathrm{H}) .{ }^{13} \mathrm{C} \mathrm{NMR}(\mathrm{CDCl}): \delta 75.6$ (t, $\left.\mathrm{OCH}_{2}\right) ; \delta 117.5\left(\mathrm{t},=\mathrm{CH}_{2}\right) ; \delta 128.8\left(\mathrm{~d}, \mathrm{C}_{3,5}\right) ; \delta 129.3\left(\mathrm{~s}, \mathrm{C}_{4}\right) ; \delta$ 133.1 (d, $=\mathrm{CH}-) ; \delta 133.2\left(\mathrm{~s}, \mathrm{C}_{26}\right) ; \delta 153.4\left(\mathrm{~s}, \mathrm{C}_{1}\right)$. MS: $\left(\mathrm{M}^{+}-41\right)$ : 363.121, Calc. 363.121. (Found: C. 55.35; H, 6.99; Cl, 8.80. Calc. for $\mathrm{C}_{19} \mathrm{H}_{28} \mathrm{O}_{7} \mathrm{Cl}: \mathrm{C}, 56.36 ; \mathrm{H}, 7.22 ; \mathrm{Cl}, 8.76 \%$ ).

4-Chloro - 2,6 - bis (hydroxyethoxyethoxymethyl) - 1 - (2 propenyloxy)phenyl mono and bis toluene - $p$ - sulphonate 14 resp. 10 were prepared from 9 according to the procedure of Pearson et al. ${ }^{26}$ Purification was accomplished by chromatography on silicagel $\left(\mathrm{CHCl}_{3}\right.$-EtOAc 6/4).

Monotosylate 14, yield 9\%. IR (neat): 925. 1100, 1180, 1190 $1355,1450,2870$ and $3490 \mathrm{~cm}^{-1}$. 'H NMR (CDCl $\left.)_{3}\right): \delta 2.17$ (br s, $\mathrm{OH}, 1 \mathrm{H}) ; \delta 2.43$ (s, $\mathrm{ArCH}_{3}, 3 \mathrm{H}$ ); $\delta 3.61$ (s, $\left.\mathrm{OCH}_{2} \mathrm{CH}_{2} \mathrm{O}, 4 \mathrm{H}\right) ; \delta$ $3.69\left(\mathrm{~s}, \mathrm{OCH}_{2} \mathrm{CH}_{2} \mathrm{O}, 8 \mathrm{H}\right) ; \delta 3.69\left(\mathrm{t}, J=3 \mathrm{~Hz}, \mathrm{OCH}_{2} \mathrm{CH}_{2} \mathrm{Tos}\right) ; \delta$ $4.18\left(\mathrm{t}, J=3 \mathrm{~Hz}, \mathrm{OCH}_{2} \mathrm{CH}_{2} \mathrm{Tos}, 2 \mathrm{H}\right) ; \delta 4.36\left(\mathrm{~d}, J=5 \mathrm{~Hz}, \mathrm{OCH}_{2}\right.$, $2 \mathrm{H}) ; \delta 4.53\left(\mathrm{~s}, \mathrm{ArCH}_{2}, 2 \mathrm{H}\right) ; \delta 4.58\left(\mathrm{~s}, \mathrm{ArCH}_{2}, 2 \mathrm{H}\right) ; \delta 5.25(\mathrm{~m}$ of d, $\left.J=10 \mathrm{~Hz},=\mathrm{CH}_{2}, 1 \mathrm{H}\right) ; \delta 5.46\left(\mathrm{~m}\right.$ of $\left.\mathrm{d}, J=16 \mathrm{~Hz},=\mathrm{CH}_{2}, 1 \mathrm{H}\right) ; \delta$ 6.10 (ddt, $J=5,10$ and $16 \mathrm{~Hz},=\mathrm{CH}-, 1 \mathrm{H}) ; \delta 7.33(\mathrm{~d}, J=7 \mathrm{~Hz}$, $\mathrm{ArH}, 2 \mathrm{H}) ; \delta 7.37(\mathrm{~s}, \mathrm{ArH}, 2 \mathrm{H}) ; \delta 7.80(\mathrm{~d}, J=7 \mathrm{~Hz}, \mathrm{ArH}, 2 \mathrm{H}) .{ }^{13} \mathrm{C}$ NMR $\left(\mathrm{CDCl}_{3}\right): \delta 75.6\left(\mathrm{t}, \mathrm{OCH}_{2}\right) ; \delta 117.4\left(\mathrm{t},=\mathrm{CH}_{2}\right) ; \delta 127.7(\mathrm{~d}$ $\left.\mathrm{ArSO}_{2}\right) ; \delta 128.7\left(\mathrm{~d}, \mathrm{C}_{3.5}\right) ; \delta 128.9\left(\mathrm{~s}, \mathrm{ArSO}_{2}\right) ; \delta 129.3\left(\mathrm{~s}, \mathrm{C}_{4}\right) ; \delta$ $129.6\left(\mathrm{~d}, \mathrm{ArSO}_{2}\right) ; \delta 132.9$ (d, $\left.=\mathrm{CH}-\right) ; \delta 133.2\left(\mathrm{~s}, \mathrm{C}_{2.6}\right) ; \delta 144.5(\mathrm{~s}$ $\mathrm{ArSO}_{2}$ ); $\delta 153.4$ (s, $\mathrm{C}_{1}$ ). MS: $\left(\mathrm{M}^{+}-41\right)$ : 517.132 , Calc. 517.130 .

Ditosylate 10, yield $58 \%$. IR (neat): $920,1000,1100,1180,1360$, 1450 and $2880 \mathrm{~cm}^{-1}$. ' $\mathrm{H}$ NMR $\left(\mathrm{CDCl}_{3}\right): \delta 2.42\left(\mathrm{~s}, \mathrm{ArCH}_{3}, 6 \mathrm{H}\right) ; \delta$ $3.61\left(\mathrm{~s}, \mathrm{OCH}_{2} \mathrm{CH}_{2} \mathrm{O}, 8 \mathrm{H}\right) ; \delta 3.67\left(\mathrm{t}, J=5 \mathrm{~Hz}, \mathrm{OCH}_{2} \mathrm{CH}_{2} \mathrm{Tos}, 4 \mathrm{H}\right)$ $\delta 4.16\left(\mathrm{t}, J=5 \mathrm{~Hz}, \mathrm{OCH}_{2} \mathrm{CH}_{2} \mathrm{Tos}, 4 \mathrm{H}\right) ; \delta 4.35(\mathrm{~d}, J=5 \mathrm{~Hz}$. $\left.\mathrm{OCH}_{2}, 2 \mathrm{H}\right) ; \delta 4.52\left(\mathrm{~s}, \mathrm{ArCH}_{2}, 4 \mathrm{H}\right) ; \delta 5.24\left(\mathrm{~d}, J=9.5 \mathrm{~Hz},=\mathrm{CH}_{2}\right.$ $1 \mathrm{H}) ; \delta 5.39\left(\mathrm{~d}, J=14.5 \mathrm{~Hz},=\mathrm{CH}_{2}, 1 \mathrm{H}\right) ; \delta 6.08(\mathrm{ddt}, J=5,9.5$ and $14.5 \mathrm{~Hz},=\mathrm{CH}-, 1 \mathrm{H}) ; \delta 7.32(\mathrm{~d}, J=8 \mathrm{~Hz}, \mathrm{ArH}, 4 \mathrm{H}) ; \delta 7.32(\mathrm{~s}$, ArH, $2 \mathrm{H}) ; \delta 7.97(\mathrm{~d}, J=8 \mathrm{~Hz}, \mathrm{ArH}, 4 \mathrm{H}) .{ }^{13} \mathrm{C} \mathrm{NMR}\left(\mathrm{CDCl}_{3}\right): \delta$ $75.8\left(\mathrm{t}, \mathrm{OCH}_{2}\right) ; \delta 117.3\left(\mathrm{t},=\mathrm{CH}_{2}\right) ; \delta 127.7\left(\mathrm{~d}, \mathrm{ArSO}_{2}\right) ; \delta 128.6(\mathrm{~d}$ $\left.\mathrm{C}_{3,3}\right) ; \delta 128.6\left(\mathrm{~s}, \mathrm{ArSO}_{2}\right) ; \delta 129.2\left(\mathrm{~s}, \mathrm{C}_{4}\right) ; \delta 129.6\left(\mathrm{~d}, \mathrm{ArSO}_{2}\right) ; \delta$ $132.8(\mathrm{~d},=\mathrm{CH}-) ; \delta 133.3\left(\mathrm{~s}, \mathrm{C}_{2,6}\right) ; \delta 144.6\left(\mathrm{~s}, \mathrm{ArSO}_{2}\right) ; \delta 153.4(\mathrm{~s}$, $\left.C_{1}\right)$.

$5^{\prime}$ - Chloro - 2' - (2 - propenyloxy) - $1^{\prime}, 3^{\prime}-x y l y l$ - dibenzo - 30 crown - 9 (12). A suspension of $2.2 \mathrm{gr}(3.1 \mathrm{mmol}) 10.0 .9 \mathrm{gr}(3.1$ $\mathrm{mmol}$ ) bis[2 - (o - hydroxyphenoxy)ethyl]ether" and $1.9 \mathrm{gr}$ (12.4 $\mathrm{mmol}) \mathrm{CsF}$ in $40 \mathrm{ml}$ acetonitrile was refluxed for $20 \mathrm{hr}$. The suspension was concentrated in vacuo, then water and $\mathrm{CHCl}_{\text {, }}$ were added. Separation of the organic layer, drying and concentration in vacuo afforded $1.74 \mathrm{gr}$ of oil, that on purification by chromatography on silicagel $\left(\mathrm{CHCl}_{3}-\mathrm{EtOH}, 5 \%\right)$, gave 12 iyield $87 \%$ ). IR (neat): $1050,1120,1200,1250,1450,1500,1590$ and $2860 \mathrm{~cm}^{-1}$. ' $\mathrm{H}$ NMR $\left(\mathrm{CDCl}_{3}\right): \delta 3.69\left(\mathrm{~s}, \mathrm{OCH}_{2} \mathrm{CH}_{2} \mathrm{O}, 8 \mathrm{H}\right) ; \delta 3.89$ (t. $\left.J=5 \mathrm{~Hz}, \mathrm{OCH}_{2} \mathrm{CH}_{2} \mathrm{O}, 8 \mathrm{H}\right) ; \delta 4.17$ (t, $J=5 \mathrm{~Hz}, \mathrm{OCH}_{2} \mathrm{CH}_{2} \mathrm{O}$, $8 \mathrm{H}) ; \delta 4.33\left(\mathrm{~m}\right.$ of d, $\left.J=5 \mathrm{~Hz}, \mathrm{OCH}_{2}, 2 \mathrm{H}\right) ; \delta 4.53\left(\mathrm{~s}, \mathrm{ArCH}_{2}, 4 \mathrm{H}\right)$; $\delta 5.20$ (m of d, $\left.J=9.5 \mathrm{~Hz},=\mathrm{CH}_{2}, 1 \mathrm{H}\right) ; \delta 5.35$ (m of d. $J=15 \mathrm{~Hz}$ $\left.=\mathrm{CH}_{2}, \mathrm{lH}\right) ; \delta 5.75-6.34(\mathrm{~m},=\mathrm{CH}-, \mathrm{IH}) ; \delta 6.90(\mathrm{~s}, \mathrm{ArH}, 8 \mathrm{H}) ; \delta$ $7.36(\mathrm{~s}, \mathrm{ArH}, 2 \mathrm{H}) .{ }^{13} \mathrm{C} \mathrm{NMR}\left(\mathrm{CDCl}_{3}\right): \delta 75.6\left(\mathrm{t}, \mathrm{OCH}_{2}\right) ; \delta 115.0$ (d. Ar); $\delta 117.3\left(\mathrm{t},=\mathrm{CH}_{2}\right) ; \delta 121.5$ (d, Ar); $\delta 128.6$ (d, $\left.\mathrm{C}_{4 . \mathrm{o}}\right) ; \delta$ $129.3\left(\mathrm{~s}, \mathrm{C}_{5}\right) ; \delta 133.3\left(\mathrm{~s}, \mathrm{C}_{13}\right) ; \delta 133.3(\mathrm{~d},=\mathrm{CH}-) ; \delta 148.9(\mathrm{~s}, \mathrm{Ar}) ; \delta$ $153.3\left(\mathrm{~s}, \mathrm{C}_{2}\right)$. MS: $\mathrm{M}^{+} 658.258$, Calc. 658.255 . 
5' - Chloro - 2' - hydroxy - 1',3' - xylyl - dibenzo - 30 - crown - 9 (13) was prepared from 12 according to the procedure of Boss and Scheffold ${ }^{17}$ with the difference that the reaction was carried out in EtOH-5\% THF. Purification was accomplished by chromatography on silicagel $\left(\mathrm{CHCl}_{3}-\mathrm{E} 1 \mathrm{OH}, 4 \%\right)$, yield $64 \%$. IR (neat): $1100,1250,1350,1450,1600,2900$ and $3340 \mathrm{~cm}^{-1}$. ${ }^{1} \mathrm{H}$ NMR $\left(\mathrm{CDCl}_{3}\right): \delta 3.70\left(\mathrm{~s}, \mathrm{OCH}_{2} \mathrm{CH}_{2} \mathrm{O}, 8 \mathrm{H}\right) ; \delta 3.88(\mathrm{t}, J=5 \mathrm{~Hz}$, $\left.\mathrm{OCH}_{2} \mathrm{CH}_{2} \mathrm{O}, 8 \mathrm{H}\right) ; \delta 4.17\left(\mathrm{t}, J=5 \mathrm{~Hz}, \mathrm{OCH}_{2} \mathrm{CH}_{2} \mathrm{O}, 8 \mathrm{H}\right) ; \delta 4.58$ (br s, $\left.\operatorname{ArCH}_{2}, 4 \mathrm{H}\right) ; \delta 6.90(\mathrm{~s}, \operatorname{ArH}, 8 \mathrm{H}) ; \delta 7.27$ (s, ArH, 2H); $\delta$ 7.81 (br s, OH, IH). ${ }^{13} \mathrm{C} \mathrm{NMR}\left(\mathrm{CDCl}_{3}\right): \delta 75.6\left(\mathrm{t}, \mathrm{OCH}_{2}\right) ; \delta 115.0$ (d, Ar); $\delta 121.5$ (d, Ar); $\delta 124.0\left(\mathrm{~s}, \mathrm{C}_{3}\right) ; \delta 125.6\left(\mathrm{~s}, \mathrm{C}_{1.3}\right) ; \delta 127.3$ (d, $\left.\mathrm{C}_{4,6}\right) ; \delta 148.8(\mathrm{~s}, \mathrm{Ar}) ; \delta 152.2\left(\mathrm{~s}, \mathrm{C}_{2}\right)$. MS: $\mathrm{M}^{+}$618.231, Calc. 618.223 .

5' - Chloro - 2' - (2 - propenyloxy) - 1',3' - xylyl - 18 - crown - 5 6a. A suspension of $414 \mathrm{mg}(0.74 \mathrm{mmol})$ monotosylate 14 and $50 \mathrm{mg}(2.2 \mathrm{mmol}) \mathrm{NaH}$ in $10 \mathrm{ml}$ THF was refluxed for $1.5 \mathrm{hr}$; then water and ether were added. The organic layer was separated, dried and concentrated in vacuo. The residue was purified by chromatography on silicagel ( $\left.\mathrm{CHCl}_{3}-\mathrm{EtOAc} 6-4\right)$, yield $151 \mathrm{mg}$ (53\%), m.p. $96-97^{\circ} \mathrm{C}$. Spectral data were in agreement with the structure. $6 a$ was obtained in $30 \%$ yield when $\mathrm{KO} \mathrm{t}-\mathrm{Bu}$ was used as the base.

Acknowledgements-The authors wish to thank Prof. D. J. Cram and Dr. R. C. Helgeson for helpfull discussions on the synthesis of crown ethers with acidic groups in general and the use of the allyl protecting group in crown ether chemistry.

\section{REFERENCES}

'Throughout this paper we have followed the trivial nomenclature that was first introduced by Pedersen ${ }^{2}$ and is now generally accepted in crown ether chemistry.

${ }^{2}$ C. J. Pedersen, J. Am. Chem. Soc. 89, 7017 (1967).

${ }^{3}$ J. M. Timko, R. C. Helgeson and D. J. Cram, Ibid. 100, 2828 (1978).
${ }^{4} M$. Newcomb, S. S. Moore and D. J. Cram, Ibid. 99. 6405 (1977).

'I. Goldberg. Acta Crvst B31. 2592 (1975).

${ }^{6} \mathrm{M}$. A. McKervey and D. L. Mulholland, J. Chem. Soc. Chem. Commun. 438 (1977).

'S. Harkema, G. J. van Hummel, K. Daasvatn and D. N Reinhoudt, Ibid. Chem. Commun., 368 (1981).

${ }^{8}$ K. Madan and D. J. Cram, Ibid. Chem. Commun. 427 (1975).

${ }^{9}$ E. P. Kyba, R. C. Helgeson, K. Madan, G. W. Gokel, T. L. Tarnowski, S. S. Moore and D. J. Cram, J. Am. Chem. Soc. 99, 2564 (1977).

${ }^{10} \mathrm{D}$. N. Reinhoudt and F. de Jong, Progress in Macrocyclic Chemistry (Edited by R. M. Izatt and J. J. Christensen), Vol. I, p. 157. Wiley, New York (1979).

"S. S. Moore, T. L. Tarnowski, M. Newcomb and D. J. Cram, J. Am. Chem. Soc. 99,6398 (1977).

${ }^{12}$ R. T. Gray, D. N. Reinhoudt, C. J. Smit and I. Veenstra, Recl. Trav. Chim. Pays-Bas 95, 258 (1976).

${ }^{13}$ F. Wada, R. Arata, T. Goto, K. Kikukawa and T. Matsuda, Bull. Chem. Soc. Jpn. 53, 2061 (1980).

${ }^{14}$ F. de Jong and D. N. Reinhoudt, Adv. Phys. Org. Chem. 17, 279 (1980).

${ }^{15}$ K. E. Koenig, G. M. Lein, P. Stuckler, T. Kaneda and D. J. Cram, J. Am. Chem. Soc. 101, 3553 (1979).

${ }^{16}$ M. E. Jung and M. A. Lyster, J. Org. Chem. 42, 3761 (1977).

${ }^{17}$ R. Boss and R. Scheffold, Angew. Chem. 88, 578 (1976).

${ }^{18}$ H. T. Openshaw and R. Robinson, J. Chem. Soc. 912 (1946).

${ }^{19} \mathrm{~J}$. Cunningham and R. Gigg, Ibid. 2968 (1965).

${ }^{20}$ D. N. Reinhoudt, F. de Jong and H. P. M. Tomassen, Tetrahedron Letters 2067 (1979).

${ }^{21} \mathrm{O}$. Piepers and R. M. Kellogg, J. Chem. Soc. Chem. Commun. 383 (1978).

${ }^{22}$ I. Goldberg, Acta Cryst B34, 3387 (1978).

${ }^{23}$ C. G. Krespan, J. Org. Chem. 39, 2351 (1974).

${ }^{24} \mathrm{M}$. Kolobielski, J. Am. Oil Chemists Soc. 45, 616 (1968); P. Rempp, Bull. Soc. Chim. France 844 (1957).

${ }^{25} \mathrm{~F}$. Vögtle and P. Neumann, Tetrahedron 26, 5299 (1970).

${ }^{26}$ D. P. J. Pearson, S. J. Leigh and I. O. Sutherland, J. Chem. Soc. Perkin I 3113 (1979). 